\title{
Giant Right Coronary Ostial Aneurysm in a Patient With Marfan Syndrome
}

\author{
Rachel Stein ${ }^{1}$, Rebekah M. Padilla ${ }^{1}$, Gregory Wynn ${ }^{1}$ \\ 1. Radiology, University of Florida College of Medicine - Jacksonville, Jacksonville, USA
}

Corresponding author: Rachel Stein, rachel.stein@jax.ufl.edu

\begin{abstract}
Aortic root dilation and aortic insufficiency are prominent causes of morbidity in Marfan syndrome. These pathologies necessitate surgical repair, including aortic root and aortic valve replacement procedures, to improve prognosis. Coronary artery aneurysms, particularly giant coronary ostial aneurysms, are rare complications of these surgeries in the Marfan population. Due to the significant life-threatening sequelae of coronary artery aneurysms, it is imperative to bring attention regarding this complication to the radiologist assessing thoracic imaging in this patient population.
\end{abstract}

Categories: Cardiac/Thoracic/Vascular Surgery, Radiology, Anatomy

Keywords: coronary artery aneurysm, marfan syndrome, ostial aneurysm, aortic root replacement, imaging

\section{Introduction}

Coronary artery aneurysms (CAAs) are defined as the segment of the artery in which dilation is 1.5 times the diameter of the adjacent segment $[1,2]$. The incidence of patients undergoing coronary angiography who are incidentally found to have CAAs is $0.2 \%-4.9 \%$ [1-3]. There are several variations of CAAs including giant coronary artery aneurysms (GCAAs), coronary ostial aneurysms, true aneurysms, and pseudoaneurysms. Most CAAs are typically found to be $<2 \mathrm{~cm}$ [4]. While there is no clear definition of GCAAs, there has been a wide range of proposed terminology including aneurysms $>20 \mathrm{~mm},>40 \mathrm{~mm},>50 \mathrm{~mm}$, four times the normal diameter, or $>4 \mathrm{~cm}$ in diameter; however, GCAAs reaching $>4 \mathrm{~cm}$ are exceedingly rare $[2,4,5]$. For the purposes of this article, we will refer to GCAAs as localized coronary artery dilations greater than $2 \mathrm{~cm}$.

GCAAs are also most often found incidentally, with an incidence of $0.02 \%$ in the general population [2]. It is imperative for the radiologist to identify CAAs as they carry the life-threatening risks of thrombosis, distal embolization, ischemia, myocardial infarction, and potential rupture [1,3]. GCAAs hold the same risks as CAAs but with a higher tendency for thrombus formation $[4,6]$. Atherosclerosis is the most common cause; however, it is known to arise in patients with vasculitides and connective tissue diseases, such as Kawasaki disease and Marfan syndrome, respectively [2,6,7]. Aortic root dilation is a major contributor to the morbidity and mortality of patients with Marfan syndrome [8,9]. Surgical repair is often necessary via the modified Bentall procedure and aortic valve-sparing root replacement, both of which markedly improve the prognosis in Marfan patients [8,10]. Given both the post-operative procedural risk and the risk of cystic

Review began 02/23/2021 Review ended 02/26/2021 Published 03/01/2021

\section{(c) Copyright 2021}

Stein et al. This is an open access article distributed under the terms of the Creative Commons Attribution License CC-BY 4.0., which permits unrestricted use, distribution, and reproduction in any medium, provided the original author and source are credited. medial necrosis, Marfan patients are at increased risk for developing GCAAs [11,12].

While reported in the literature, CAAs and GCAAs following aortic root procedures are a rare complication [2,3,6-8,11-13]. Herein we discuss a case report of a patient with Marfan syndrome who developed a giant right coronary osital aneurysm 18 years after undergoing the modified Bentall procedure.

\section{Case Presentation}

A 35-year-old male with a past medical history of Marfan syndrome and hypertension underwent aortic root replacement with a mechanical composite valve graft, $27 \mathrm{~mm}$ Carbomedics valve, 18 years prior to presentation. The patient was being treated with warfarin for mechanical valve anticoagulation. He gave a family history significant for Marfan syndrome in both his brother and father. A $2.2 \mathrm{~cm}$ giant right coronary ostial aneurysm was incidentally discovered two years prior to presentation on a CT pulmonary angiogram obtained for suspected pulmonary embolism in the setting of shortness of breath. At that time, the patient was aware of this finding, due to prior imaging at an outside hospital. Repeat CT pulmonary angiogram performed approximately 14 months later, for dyspnea on exertion, revealed an increase in the aneurysm diameter to $2.6 \mathrm{~cm}$.

On this encounter, he presented for evaluation of the right GCAA, with the primary service requesting imaging as workup prior to surgical repair of this aneurysm. A CTA of the coronary arteries with retrospective electrocardiographic gating was performed on a Siemens 64 array multidetector computed tomography scanner (Siemens, Germany). The patient's heart rate ranged from 58 to 75 beats per minute during the scan, with $0.4 \mathrm{mg}$ sublingual nitroglycerin given immediately prior to image acquisition for 


\section{Cureus}

coronary artery vasodilation. Multiplanar reconstruction, dynamic loop-imaging, and maximum intensity projections were utilized. The images demonstrated the right GCAA was found to have increased to $3.1 \mathrm{~cm}$ in diameter. The aneurysm originated from the right coronary artery ostium extending $3.5 \mathrm{~cm}$ along the course of the right coronary artery (Figures $1-4$ ).

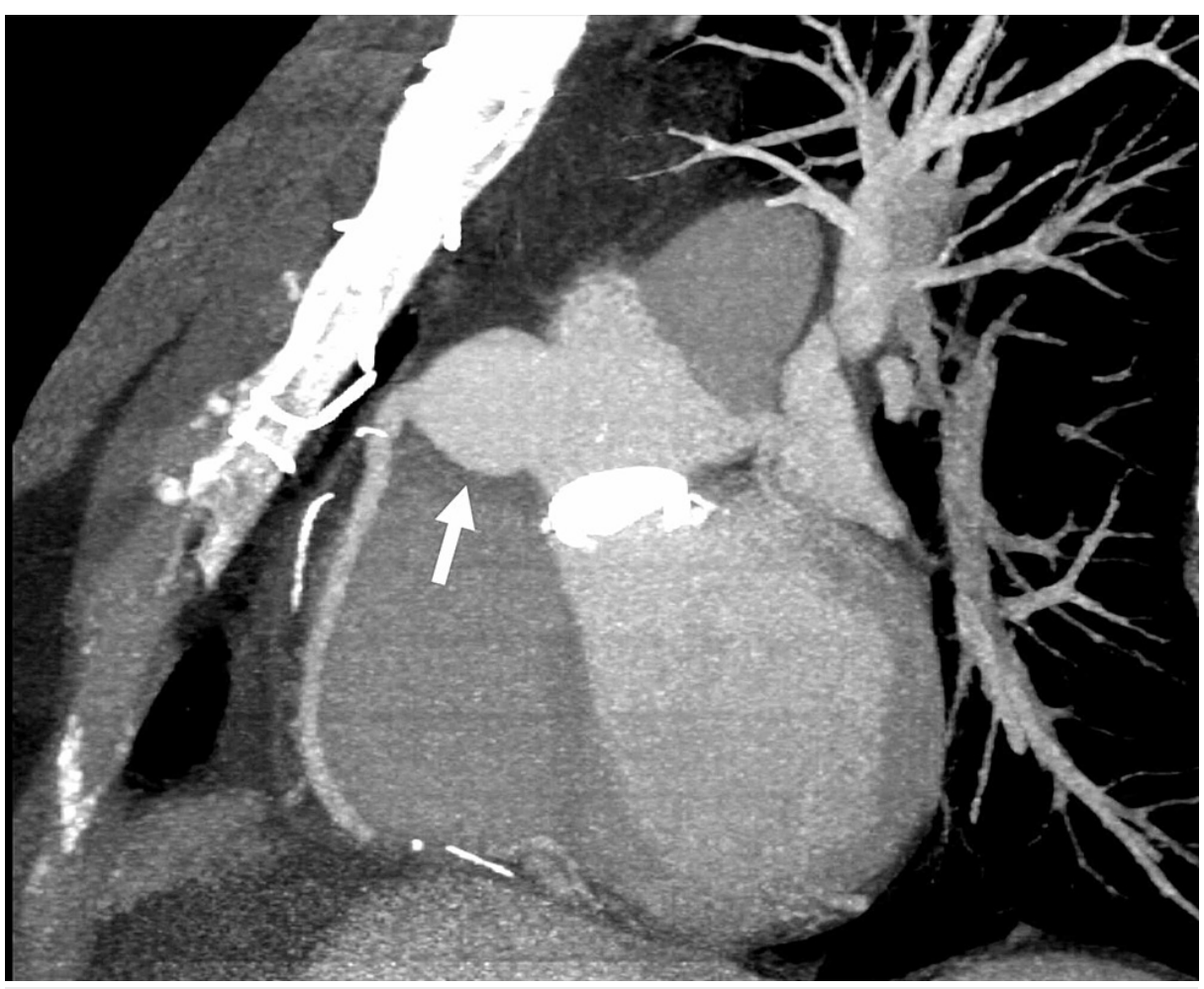

FIGURE 1: Maximum Intensity Projection Sagittal CTA Image

Origin of the right coronary artery with aneurysmal dilatation of the first $3.5 \mathrm{~cm}$ of the right coronary artery, which measures as much as $3.1 \mathrm{~cm}$ in diameter (white arrow).

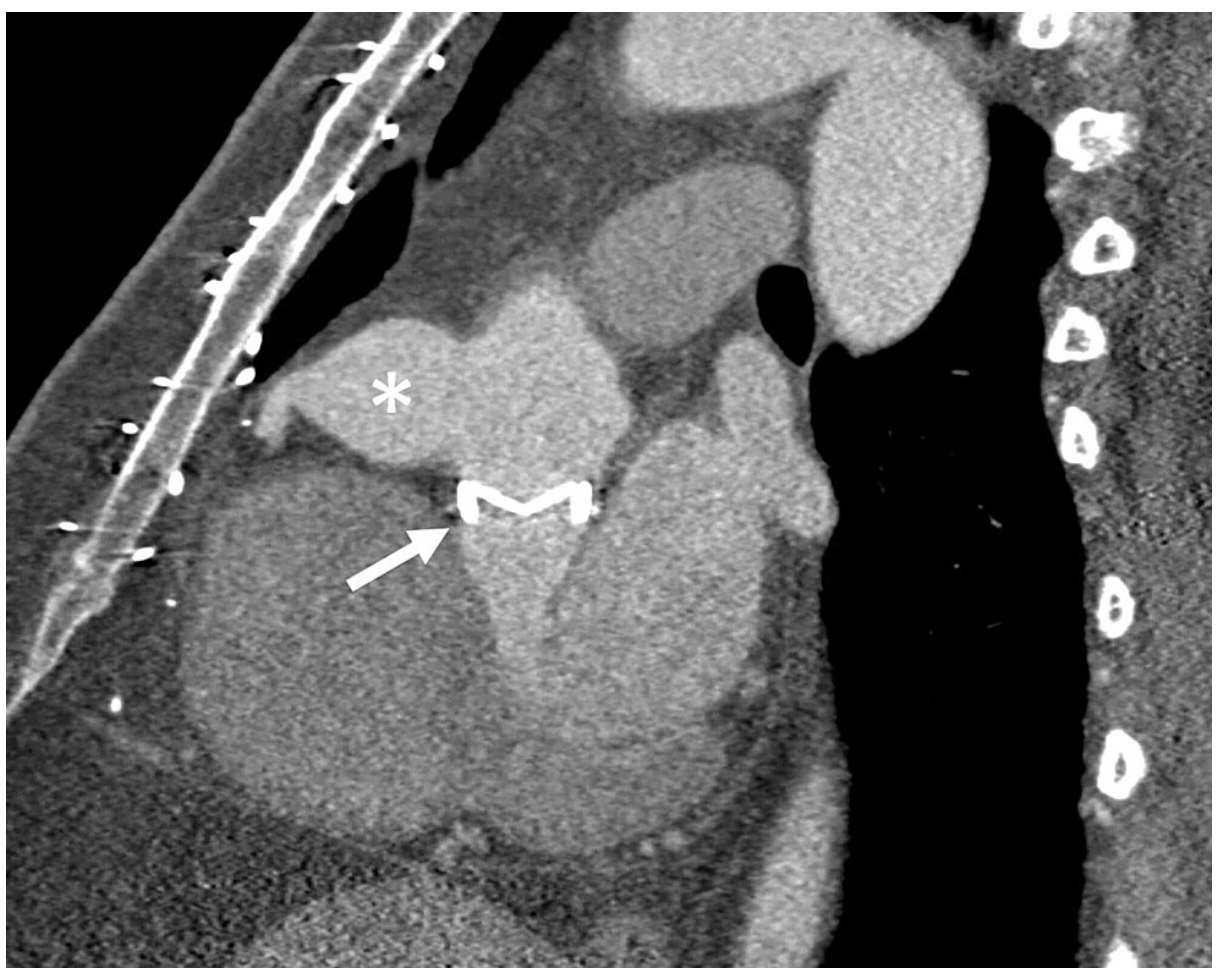

FIGURE 2: Multiplanar Reconstruction Sagittal CTA Image 


\section{Cureus}

Prosthetic aortic valve replacement (Carbomedics $27 \mathrm{~mm}$ valve) (white arrow) and the aneurysm of the proximal right coronary artery and ostial dilatation (asterisk).

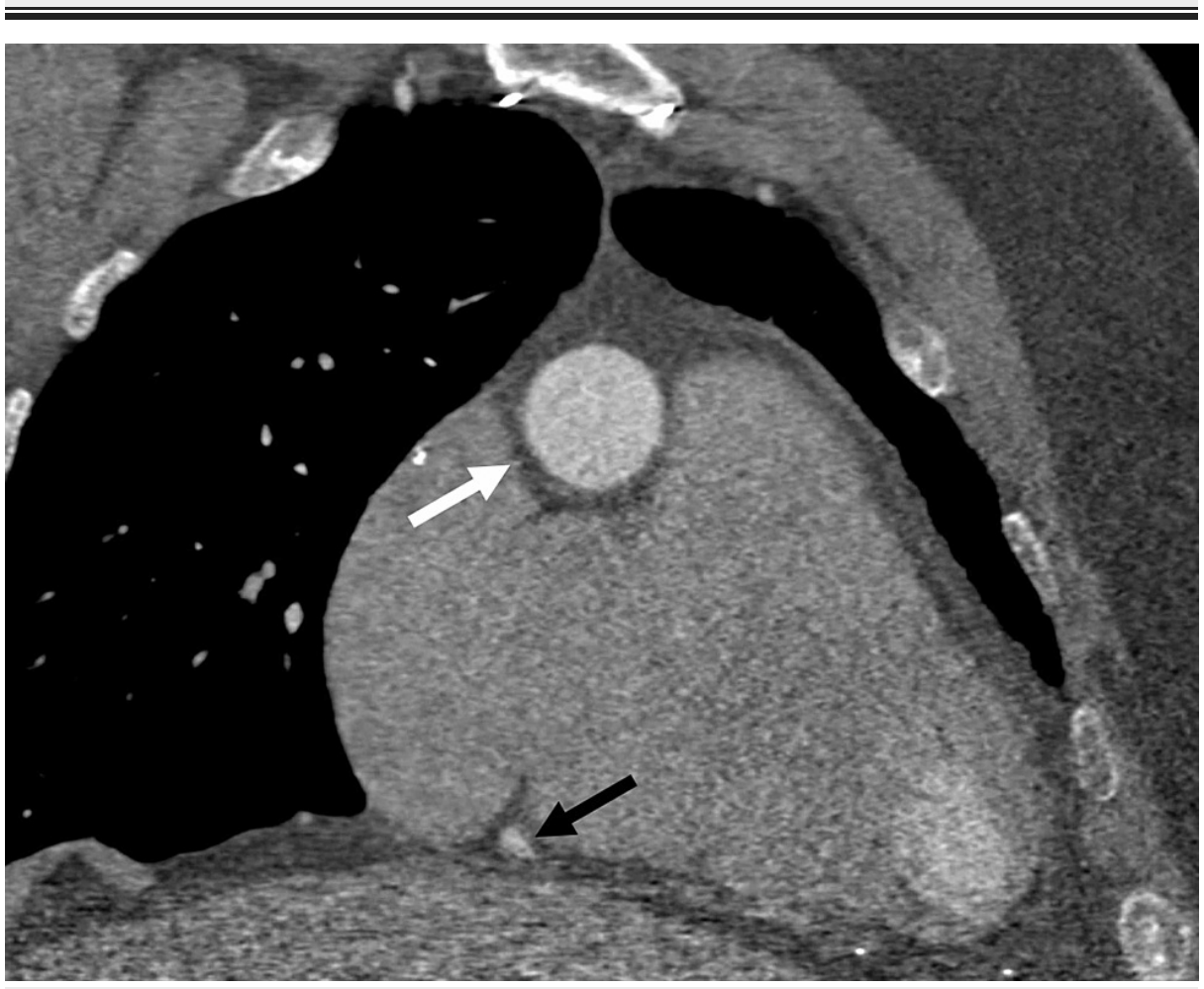

FIGURE 3: Multiplanar Reconstruction Oblique Coronal CTA Image

Right coronary artery demonstrates aneurysmal dilatation of the artery just distal to the ostium (white arrow). The caliber of the distal right coronary artery is normal (black arrow). 


\section{Cureus}

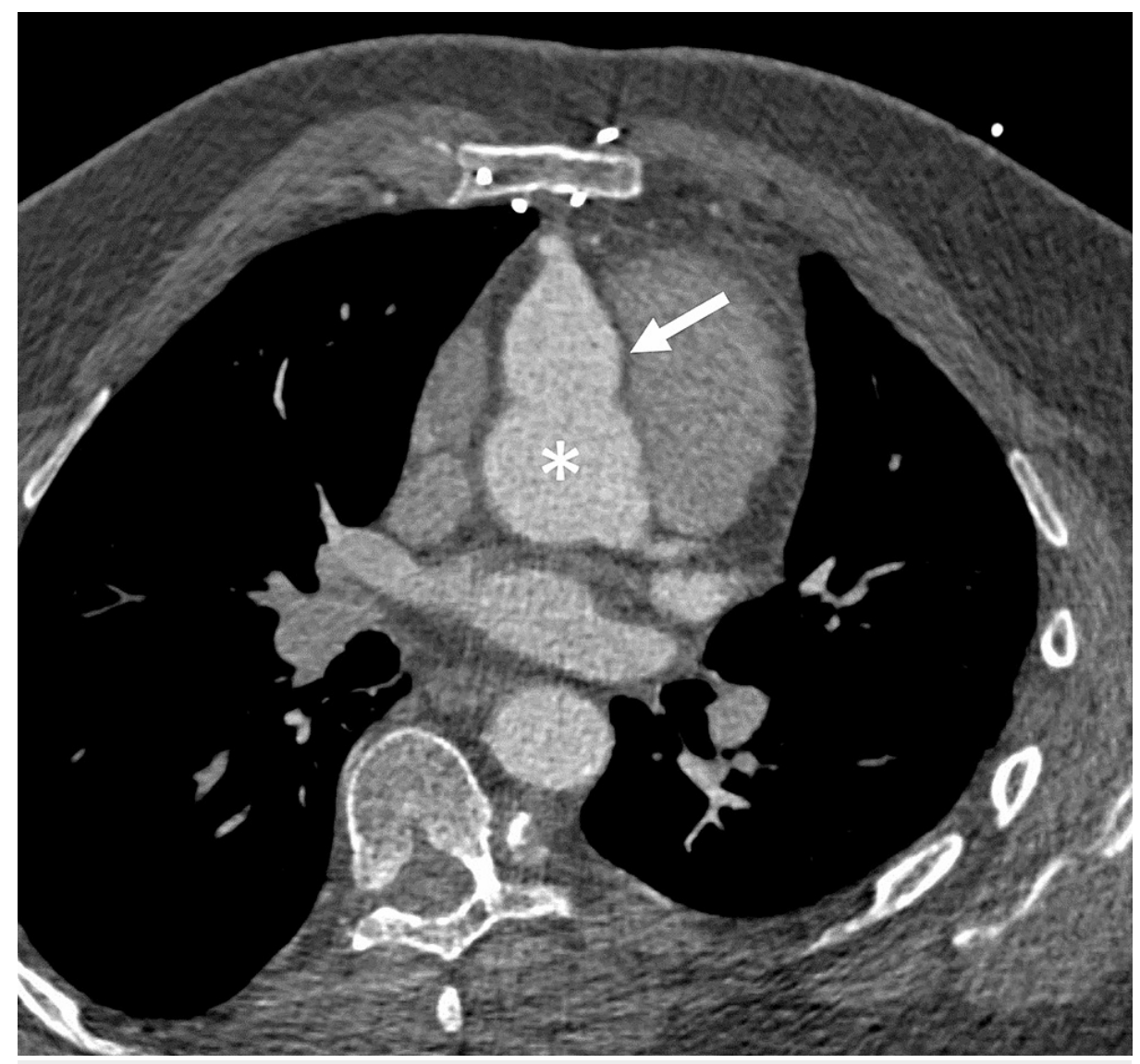

\section{FIGURE 4: Multiplanar Reconstruction Axial CTA Image}

Ostial dilation and proximal aneurysm of the right coronary artery (white arrow) approaching the diameter of the proximal aorta (asterisk).

\section{Discussion}

Marfan syndrome is an autosomal dominant defect in fibrillin that causes a systemic disorder of connective tissue disease and is the most common genetic cause of aortic deficiency $[3,8,9]$. Aortic root dilation and aortic valve insufficiency account for the major morbidity and mortality associated with Marfan syndrome [9]. The modified Bentall procedure and aortic valve-sparing root surgery are two options for surgical management that have significantly improved the prognosis for Marfan patients with aortic root dilation $[8,10,11,14]$. Coronary ostial aneurysms are rare complications in Marfan patients following the modified Bentall procedure [2,3,6-8,11-13]. Knowledge of regional anatomy and the surgical technique utilized helps in understanding why these aneurysms occur in this patient population.

The aortic root connects the left ventricle to the ascending aorta and is comprised of the aortic annulus, sinuses of Valsalva, and sinotubular junction [15]. The coronary ostia arise from the dilated sinuses of Valsalva, which function to prevent occlusion of the ostia during systole and aortic valvular opening [16]. In both the modified Bentall procedure and aortic valve-sparing root surgery, the native coronary arteries are removed with a cuff of adventitia and re-implanted onto the aortic root graft, predisposing to wall weakness and possible aneurysm formation $[8,13]$. Marfan patients are also believed to be at increased risk for developing CAAs from cystic medial necrosis, which causes weakening of the tunica media in the vessel wall $[2,12]$.

In the review of the literature, there are several types of CAAs that can occur after these procedures of which the radiologist should be aware. Definitions and measuring parameters for each type of aneurysm may be found in Table 1. As a radiologist, it is essential to understand the differences in aneurysm variations as treatment differs between them. The first step in defining the type of aneurysm is identifying whether the aneurysm is a true aneurysm or a pseudoaneurysm. On CTA, a true aneurysm will show contrast contained within the arterial lumen as compared to a pseudoaneurysm that demonstrates an extraluminal collection of contrast and may have a thin neck connecting the aneurysm to the involved coronary artery [8]. Most CAAs are $<2 \mathrm{~cm}$ in diameter [4]. Our patient presented with a $2.2 \mathrm{~cm}$ aneurysm that progressed to $2.6 \mathrm{~cm}$ over a 14 month period and further increased to $3.1 \mathrm{~cm}$ over an additional 10 months. As alluded to earlier, there is wide variation in the definition of GCAAs; thus, we chose to label our patient's aneurysm as a GCAA since it 


\section{Aneurysm}

True aneurysm

Pseudoaneurysm (false aneurysm)

Coronary artery aneurysm

Giant coronary artery aneurysm

Coronary ostial aneurysm

Coronary button ostial aneurysm

\section{Definition}

Fusiform or saccular dilation of entire vessel wall

Rupture or perforation of artery encased by blood clot or tunica adventitia

Localized dilation of 1.5 times or $50 \%$ more than the diameter of the adjacent segment

Aneurysm $>2 \mathrm{~cm}$ to $>4 \mathrm{~cm}$ in diameter

$>0.1 \mathrm{~cm}$ at the coronary ostial reimplantation site

$>0.1 \mathrm{~cm}$ dilation of the "button" of native aorta at the ostia reimplantation site

TABLE 1: Types of Coronary Artery Aneurysms and Their Definitions

While there is no clearly defined imaging protocol post-surgery, patients may undergo follow up with various imaging modalities, such as transthoracic ultrasound, CTA, magnetic resonance angiography (MRA), or coronary angiography [8]. Groner et al. state that CTA and MRA are the main surveillance imaging modalities of the post-surgical aorta in Marfan syndrome [8]. Electrocardiogram (ECG)-gated CTA has emerged to the forefront in assessing coronary artery anomalies and acute aortic diseases [17,18]. Earlier case reports of CAAs in Marfan patients post-surgery were diagnosed via non-gated CTA, whereas more recent reports, including our report, have diagnosed CAAs via ECG-gated CTA [7,11]. The benefit of ECGgated over conventional non-gated CTA is the ability to decrease artifact by reducing aortic root motion, thereby improving the resolution of the coronary arteries and increasing diagnostic accuracy $[11,18]$. Often, invasive imaging with coronary angiography is recommended to further characterize the aneurysm's size, shape, and specific location [2]. However, it has been suggested that ECG-gated CTA may be superior to coronary angiography [19].

Regardless of the imaging modality used, potential aneurysmal dilatation of the coronary arteries should be considered by the radiologist when assessing studies obtained in the Marfan population who have undergone previous aortic root procedures. Appreciation of the life-threatening risks of thrombosis, distal embolization, ischemia, myocardial infarction, and potential rupture associated with these aneurysms is also necessary $[2,3]$. Depending on the size of the coronary aneurysm, treatment modalities include medical management with anticoagulation, stenting, and coil embolization. Surgical repair is indicated if the aneurysm is three times the diameter of the original vessel [5]. Given the potential for a life-threatening rupture, GCAAs should receive surgical repair [12].

\section{Conclusions}

Patients with Marfan syndrome are at increased risk of developing giant coronary ostial aneurysms following aortic root and valve replacement surgery. While no strict imaging protocol has been defined following these operative procedures in Marfan patients, ECG-gated CTA has emerged over time as the study of choice for coronary artery assessment. Defining GCAAs accurately is important as they carry a higher risk for thrombosis and necessitate surgical repair to prevent rupture.

\section{Additional Information}

\section{Disclosures}

Human subjects: Consent was obtained or waived by all participants in this study. Conflicts of interest: In compliance with the ICMJE uniform disclosure form, all authors declare the following: Payment/services info: All authors have declared that no financial support was received from any organization for the submitted work. Financial relationships: All authors have declared that they have no financial relationships at present or within the previous three years with any organizations that might have an interest in the submitted work. Other relationships: All authors have declared that there are no other relationships or activities that could appear to have influenced the submitted work.

\section{References}

1. Chrissoheris MP, Donohue TJ, Young RS, et al.: Coronary artery aneurysms. Cardiol Rev. 2008, 16:116-23. 10.1097/CRD.0b013e31815d0573

2. Pham V, Hemptinne Q, Grinda JM, et al.: Giant coronary aneurysms, from diagnosis to treatment: a literature review. Arch Cardiovasc Dis. 2020, 113:59-69. 10.1016/j.acvd.2019.10.008

3. Badmanaban B, Mallon P, Campbell N, et al.: Repair of left coronary artery aneurysm, recurrent ascending aortic aneurysm, and mitral valve prolapse 19 years after Bentall's procedure in a patient with Marfan 
syndrome. J Card Surg. 2004, 19:59-61. 10.1111/j.0886-0440.2004.02052.x

4. Chalkley RA, Roberts WC, Patlolla S, et al.: Giant right coronary artery aneurysms. Am J Cardiol. 2020, 125:1599-601. 10.1016/j.amjcard.2020.02.008

5. Nichols L, Lagana S, Parwani A: Coronary artery aneurysm: a review and hypothesis regarding etiology . Arch Pathol Lab Med. 2008, 132:823-8. 10.1043/1543-2165(2008)132[823:CAAARA]2.0.CO;2

6. Beckmann E, Rustum S, Marquardt S, et al.: Surgical treatment of coronary artery aneurysms. J Card Surg. 2017, 32:674-9. 10.1111/jocs.13227

7. Onoda K, Tanaka K, Yuasa U, et al.: Coronary artery aneurysm in a patient with Marfan syndrome . Ann Thorac Surg. 2001, 72:1374-7. 10.1016/s0003-4975(00)02707-7

8. Groner LK, Lau C, Devereux RB, et al.: Imaging of the postsurgical aorta in Marfan syndrome. Curr Treat Options Cardiovasc Med. 2018, 20:80. 10.1007/s11936-018-0675-2

9. Judge DP, Dietz HC: Marfan's syndrome. Lancet. 2005, 366:1965-76. 10.1016/S0140-6736(05)67789-6

10. Miyahara S, Okita Y: Overview of current surgical strategies for aortic disease in patients with Marfan syndrome. Surg Today. 2016, 46:1006-18. 10.1007/s00595-015-1278-0

11. Haroun RR, Chu LC, Fishman EK: Prevalence and natural history of coronary ostial aneurysms in Marfan patients. J Comput Assist Tomogr. 2019, 43:115-8. 10.1097/RCT.0000000000000799

12. Okamoto K, Casselman FP, De Geest R, et al.: Giant left coronary ostial aneurysm after modified Bentall procedure in a Marfan patient. Interact Cardiovasc Thorac Surg. 2008, 7:1164-6. 10.1510/icvts.2008.183103

13. Milano AD, Pratali S, Mecozzi G, et al.: Fate of coronary ostial anastomoses after the modified Bentall procedure. Ann Thorac Surg. 2003, 75:1797-801. 10.1016/s0003-4975(03)00015-8

14. Matalanis G, Perera NK: Aortic valve sparing root surgery for Marfan syndrome. Ann Cardiothorac Surg. 2017, 6:682-91. 10.21037/acs.2017.11.05

15. Loukas M, Bilinsky E, Bilinsky S, et al.: The anatomy of the aortic root. Clin Anat. 2014, 27:748-56. 10.1002/ca.22295

16. Bass D, Tivakaran VS: Sinus of Valsalva aneurysm. StatPearls Publishing, Treasure Island, FL; 2020.

17. Li Y, Fan Z, Xu L, et al.: Prospective ECG-gated 320-row CT angiography of the whole aorta and coronary arteries. Eur Radiol. 2012, 22:2432-40. 10.1007/s00330-012-2497-Z

18. Schlösser FJ, Mojibian HR, Dardik A, et al.: Simultaneous sizing and preoperative risk stratification for thoracic endovascular aneurysm repair: role of gated computed tomography. J Vasc Surg. 2008, 48:561-70. 10.1016/j.jvs.2008.04.055

19. Kim SY, Seo JB, Do KH, et al.: Coronary artery anomalies: classification and ECG-gated multi-detector row CT findings with angiographic correlation. Radiographics. 2006, 26:317-33. 10.1148/rg.262055068 